\title{
Análise do modo distorcional de elementos perfurados segundo Método da Resistência Direta
}

\author{
Luiz Henrique de Almeida Neiva ${ }^{1}$, Amaury Eloy C. Araújo Sales ${ }^{2}$, Guilherme \\ Cássio Elias ${ }^{1}$, Arlene Maria Cunha Sarmanho ${ }^{1 *}$, Flávio Teixeira de Souza ${ }^{3}$ \\ ${ }^{1}$ Programa de Pós-Graduação em Engenharia Civil - PROPEC - DECIV/EM/UFOP, \\ Ihalmeidaneiva@gmail.com,gc.elias@yahoo.com.br, \\ arlene.sarmanho@gmail.com \\ ${ }^{2}$ Mestrado Profissional em Construção Metálica - MECOM - DECIV/EM/UFOP, \\ amaurysales@gmail.com \\ ${ }^{3}$ Departamento de Edificações - IFMG, \\ flavio.souza@ifmg.edu.br
}

\section{Analysis of the distortional buckling mode of perforated elements using the Direct Strength Method}

\section{Resumo}

Este trabalho apresenta um estudo teórico e numérico da influência de perfurações no comportamento e capacidade resistente de pilares em perfis formados a frio submetidos a compressão. Pilares com diferentes seções transversais e perfurações foram avaliados numericamente via ANSYS e GBTul, com ênfase no modo de instabilidade distorcional. As forças últimas da análise numérica foram comparadas às equações do Método da Resistência Direta (MRD) da ABNT NBR 14762:2010. Finalmente, foram propostas adequações às equações do MRD para possibilitar o dimensionamento de pilares que apresentam seções com a tipologia de perfurações estudadas.

Palavras-chave: instabilidade estrutural, perfil formado a frio, instabilidade distorcional.

\begin{abstract}
This work presents a theoretical and numerical study on the influence of perforations on the behavior and strength of cold-formed steel columns subjected to compression. Members of different cross-sections and perforations were evaluated numerically via ANSYS and GBTul, with emphasis on distortional buckling mode. The ultimate loads of the numerical analysis were compared to the Direct Strength Method (DSM) equations of ABNT NBR 14762:2010. Finally, changes were proposed in the DSM equations so it is possible calculate the predicted compressive strengths of columns with perforated cross-sections studied.
\end{abstract}

Keywords: structural instability, cold-formed steel, distortional buckling. 


\section{Introdução}

Levando-se em consideração os avanços tecnológicos e fatores econômicos, os projetos de estruturas na indústria da construção civil caminham para concepção de estruturas cada vez mais leves e esbeltas. Somada ao aumento de esbeltez dos elementos, a utilização de materiais com maior resistência mecânica intensifica os fenômenos de instabilidade nessas estruturas. Tais fenômenos apresentam grande influência no comportamento e dimensionamento dos elementos.

Aos perfis obtidos a partir do perfilamento ou dobragem de chapas de aço, dá-se o nome de perfis formados a frio (PFF). Estas chapas de aço possuem características mecânicas que proporcionam a trabalhabilidade necessária ao processo de conformação. Em função dessas características, tais perfis apresentam grande variedade de seções transversais, versatilidade de aplicação, bem como reduzido peso de seus elementos quando comparados aos perfis laminados e soldados.

Em geral nos perfis em PFF são utilizadas chapas finas e as relações de largura-espessura (esbeltez local) elevadas, tornando-os mais suscetíveis aos fenômenos de instabilidade local de placa.

Pode-se entender os perfis formados a frio como uma associação de placas que compõe a seção transversal do perfil. O estudo do comportamento de placas esbeltas depende da solução de equações de equilíbrio complexas e de difícil utilização em prescrições normativas. Portanto, as normas fazem uso de métodos simplificados de solução tais como o Método da Largura Efetiva (von Karman, 1932 e Winter, 1947 apud Sarmanho, 1991); o Método da Resistência Direta (Schafer, 2006); e o Método da Seção Efetiva (Batista, 2010) cuja formulação é prevista apenas na norma brasileira ABNT NBR 14762:2010 - Dimensionamento de estruturas de aço constituídas por perfis formados a frio.

A instabilidade local de placa é caracterizada pela perda de estabilidade na qual as paredes constituintes da seção transversal tem suas formas alteradas apresentando deslocamentos laterais senoidais, mas mantendo os ângulos entre tais paredes inalterados, como observado na Figura 1. 


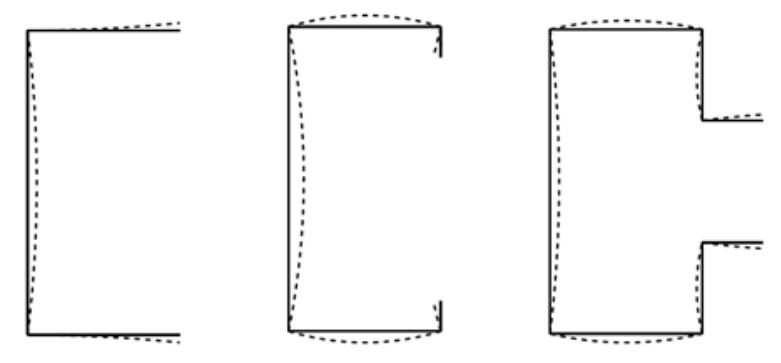

Figura 1 - Instabilidade local em seções U simples, U enrijecido e Rack (ou garrafa).

O modo de instabilidade distorcional, característico dos perfis formados a frio de seção aberta e monossimétrica, se caracteriza quando a perda de estabilidade provoca alterações na seção transversal com deslocamentos de pelo menos um dos vértices, causando abertura ou fechamento da seção, como visto na Figura 2.
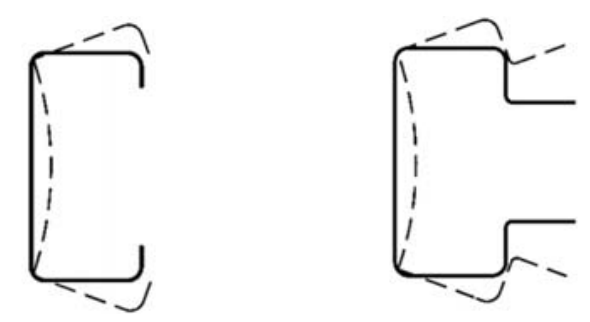

Figura 2 - Instabilidade distorcional em seções U enrijecido e Rack (Fonte: ABNT NBR 14762:2010).

Para a instabilidade global de elementos comprimidos, como os pilares, o modo se caracteriza pela perda de estabilidade em função de deslocamentos laterais ao longo do comprimento do elemento. Tal instabilidade pode ser de três tipos: por flexão em torno de um dos eixos da seção transversal; por torção em torno do eixo longitudinal; ou por flexo-torção.

Os sistemas de armazenagem industrial são estruturas utilizadas com intuito de armazenar produtos e comumente são conhecidos por racks. Os mesmos variam de pequenas estantes carregadas manualmente até estruturas com mais de $30 \mathrm{~m}$ de altura, com carregamento automatizado, os autoportantes (Godley, 1991). Na Figura 3 são representados exemplos desses sistemas. 

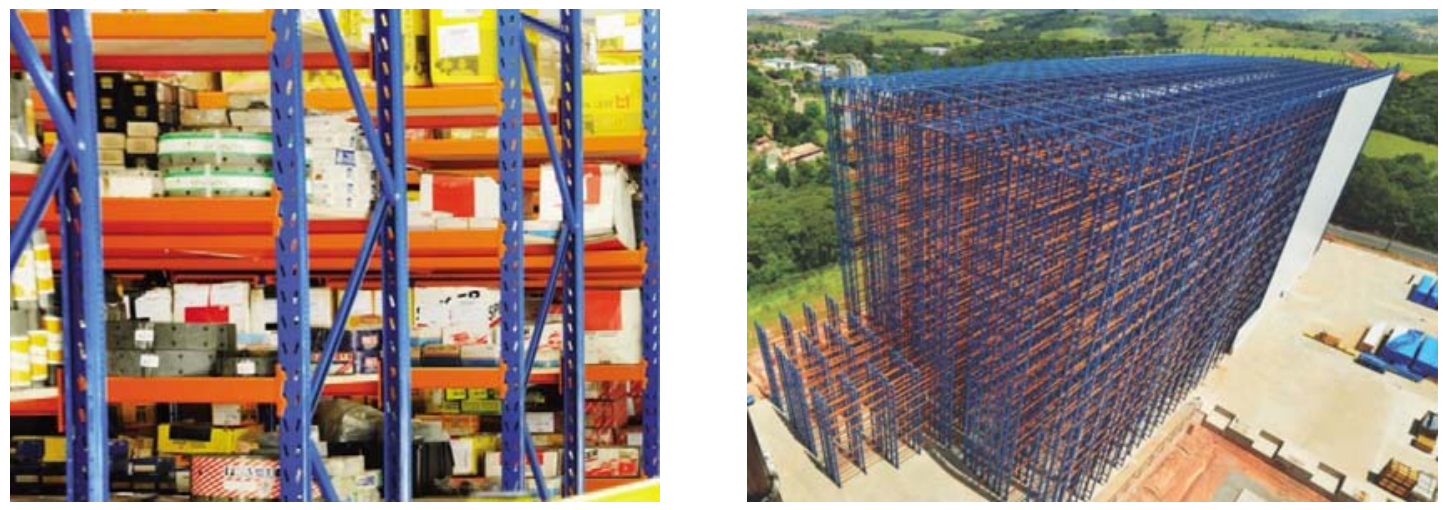

Figura 3 - Sistemas de armazenagem industrial. (Fonte: Águia, 2017).

Geralmente tais sistemas são fabricados em perfis formados a frio, com seções especialmente projetadas para facilitar sua montagem, apresentando perfurações ao longo de seu comprimento de forma a conectar as ligações. Portanto, devido a utilização de perfis (PFF) de seção esbelta, os elementos do sistema está sujeito aos fenômenos de instabilidade anteriormente citados. Adicionalmente, as perfurações existentes ao longo do comprimento dos elementos modificam o comportamento estrutural e a capacidade de carga da estrutura.

A seção comumente utilizada para os pilares desses sistemas é a tipo rack ou garrafa (Figura 4a). A existência dos flanges de ligação permite que a fixação dos contraventamentos seja diretamente no perfil, descartando a necessidade de novos elementos. Na Figura 4b observa-se um exemplo da ligação das vigas (longarinas) e contraventamentos aos pilares desses sistemas.

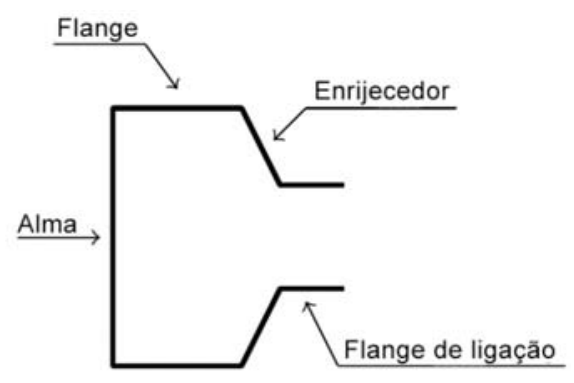

(a)

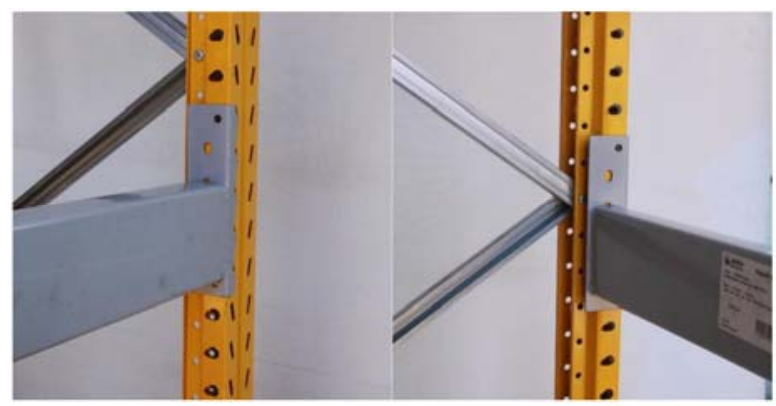

(b)

Figura 4 - Seção tipo rack (a) e fixação dos elementos (b). (Fonte: Neiva, 2017)

Estudos da influência das perfurações na estabilidade e força última de elementos comprimidos são relativamente recentes, a exemplo dos trabalhos de Schafer (2006, 2008), Souza (2013), Faria et al. (2015), Casafont et al., (2009, 2012, 2014 e 2016), Faria 
(2016) e também trabalhos relativos à utilização do Método da Resistência Direta em tais elementos, como Camotim e Basaglia (2014), Landesmann et al. (2016), Zhao et al. (2017), dentre outros.

\section{Análise teórica}

A ABNT NBR 14762:2010 permite a determinação da capacidade resistente dos elementos considerando-se o Método da Largura Efetiva, Método da Seção Efetiva ou o Método da Resistência Direta (MRD). O princípio básico deste último é a obtenção das forças últimas da seção a partir de uma análise de estabilidade elástica da seção transversal. Para tanto, é necessário resolver um problema de autovalor, determinando assim os valores das forças críticas de instabilidade. A partir dessas forças, utilizando-se as equações propostas pelo MRD, é possível determinar os valores das forças últimas.

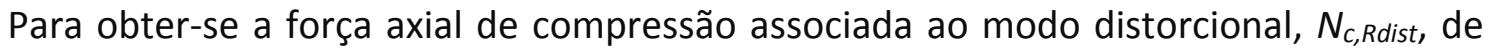
seções sem perfurações, a ABNT NBR 14762:2010 utiliza as seguintes expressões:

$$
\begin{aligned}
& \lambda_{\text {dist }} \leq 0,561 \rightarrow N_{c, \text { Rdist }}=P_{y}=A f_{y} \\
& \lambda_{\text {dist }}>0,561 \rightarrow N_{c, \text { Rdist }}=\left(1-\frac{0,25}{\lambda_{\text {dist }}^{1,2}}\right) \frac{A f_{y}}{\lambda_{\text {dist }}^{1,2}} \\
& \lambda_{\text {dist }}=\left(\frac{A f_{y}}{N_{\text {dist }}}\right)^{0,5}
\end{aligned}
$$

Onde $P_{y}$ é a força axial de escoamento; $A$ é a área bruta da seção transversal; $f_{y}$ é a tensão de escoamento do aço; $N_{\text {dist }}$ é a força axial de instabilidade distorcional elástica; e $\lambda_{\text {dist }}$ é o índice de esbeltez reduzido associado à instabilidade distorcional.

\subsection{Método da Resistência Direta para seções com perfurações}

Moen e Schafer (2008) realizaram uma análise teórico experimental de perfis U enrijecido com perfurações de forma a propor modificações no Método da Resistência Direta, a fim de que o mesmo pudesse ser utilizado no dimensionamento de seções com furos. O trabalho é abrangente, considerando elementos submetidos à compressão e flexão, e aos três modos de instabilidade: local, distorcional e global. No que concerne à compressão, alguns limites de dimensões relacionados aos furos (Equações 2.2) e chapas foram impostos para a melhor convergência do método proposto. 


$$
\frac{S}{h} \geq 1,5 \quad \frac{S}{L_{\text {hole }}} \geq 2,0 \quad \frac{L_{\text {hole }}}{h} \leq 0,75
$$

Em que $S$ é a distância longitudinal entre os furos, $h$ é a largura da chapa em que o furo está inserido e $L_{\text {hole }}$ é a altura do furo.

O recorte do presente estudo, no entanto, são os pilares submetidos à compressão uniforme em que a instabilidade distorcional seja dominante.

A proposta de Moen e Schafer (2008) é a da obtenção das forças críticas de instabilidade via Método das Faixas Finitas, considerando a utilização de uma espessura fictícia que considere a influência da perfuração no comportamento dos pilares. Assim sendo, a espessura fictícia da alma com perfurações é dada por:

$$
t_{\text {web hole }}=\left(1-\frac{L_{h}}{L_{c r d}}\right)^{1 / 3} t_{w}
$$

Onde $t_{w e b, h o l e}$ é a espessura fictícia da alma; $t_{w}$ é a espessura real da alma; $L_{h}$ é o comprimento do furo; e $L_{c r d}$ é o comprimento da meia onda do modo distorcional.

A partir dos valores de forças críticas obtidos com a espessura fictícia da alma, utiliza-se a equações do MRD de forma a serem obtidas as forças últimas dos pilares.

Com o estudo citado, Moen e Schafer (2008) propuseram modificações nas expressões originais do MRD para a obtenção da capacidade resistente de seções com perfurações. Seguindo as recomendações de Sales (2017), para as perfurações verticais estudadas neste trabalho, será utilizada a modificação das equações do MRD proposta por Moen e Schafer (2008), aqui denominadas de MOEN, dadas por:

$$
\begin{aligned}
& \lambda_{\text {dist }} \leq 0,561 \rightarrow N_{c, \text { Rdist }}=A_{\text {min, }, \text { liq }} f_{y} \\
& \lambda_{\text {dist }}>0,561 \rightarrow N_{c, \text { Rdist }}=\left(1-\frac{0,25}{\lambda_{\text {dist }}^{1,2}}\right) \frac{A_{\text {min }, \text { liq }} f_{y}}{\lambda_{\text {dist }}^{1,2}} \\
& \lambda_{\text {dist }}=\left(\frac{A_{\text {min }, \text { liq }} f_{y}}{N_{\text {dist }}}\right)^{0,5}
\end{aligned}
$$

Onde $A_{\text {min,liq }}$ é a área líquida da seção transversal considerando as perfurações. 


\section{Análise numérica}

Para a análise de estabilidade de seções em perfis formados a frio existem alguns métodos bastante difundidos, como a Teoria Generalizada de Vigas, a GBT, utilizando o software GBTul (Bebiano et al., 2008) e o Método das Faixas Finitas, via programa CuFSM (Schafer e Àdani, 2006). Todavia, os métodos anteriormente citados não permitem a avaliação de seções que apresentem perfurações, motivo pelo qual, neste trabalho foi utilizado o Método dos Elementos Finitos via o programa ANSYS (Ansys, 2016) e uma adaptação da análise via GBTul para consideração das perfurações com base nos estudos de Sales (2017).

\subsection{Geometria e identificação dos modelos}

Foram estudadas 16 seções transversais tipo rack, variando-se a largura da alma, $b_{w}$, (80 $\mathrm{mm}, 94 \mathrm{~mm}, 110 \mathrm{~mm}$ e $120 \mathrm{~mm})$ e a espessura da chapa, $t,(1,4 \mathrm{~mm}, 1,8 \mathrm{~mm}, 2,25 \mathrm{~mm}$ e 3,0 mm), como indicado na Figura 5. Também foram considerados quatro tipologias de perfurações verticais (com alturas de furos variando de $10 \%$ a $40 \%$ da largura da alma, $\left.b_{w}\right)$, gerando um total de 64 modelos.

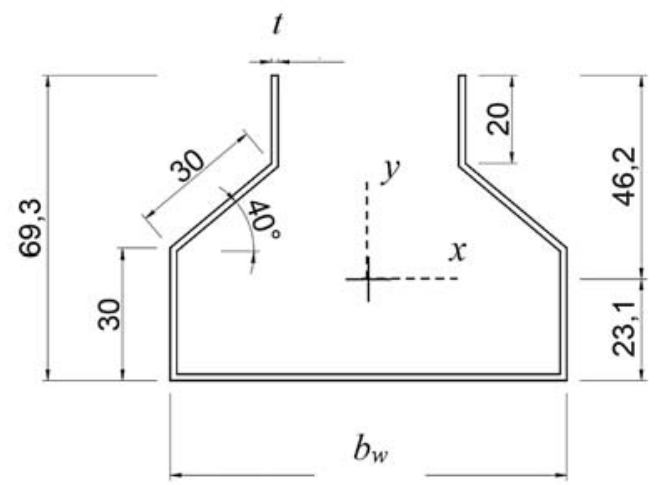

Figura 5 - Dimensões da seção transversal (em milímetros).

Para facilitar a identificação dos modelos analisados, utilizou-se uma nomenclatura que considera-se a variação da geometria das seções, por exemplo: o modelo CF-80-1,4F10\% representa um pilar com furos, largura de alma, $b_{w}$, igual a $80 \mathrm{~mm}$, espessura, $t$, de $1,4 \mathrm{~mm}$ e altura de furo, $h_{f}$, igual a $10 \%$ de $b_{w}$.

Com relação às perfurações, na Figura 6 tem-se as dimensões e espaçamentos utilizados. Tais valores foram escolhidos de modo a fornecer uma correlação entre a 
perfuração e a largura da alma, proporcionando uma melhor adequação da malha utilizada nas análises.

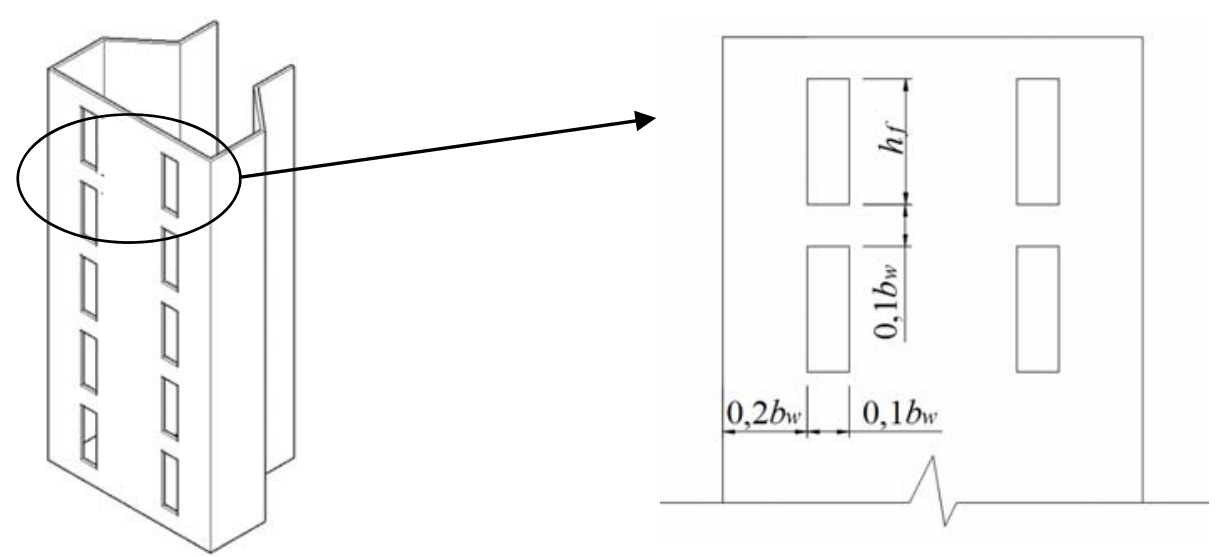

Figura 6 - Dimensões e espaçamento das perfurações.

Na Figura 7 apresenta-se as quatro tipologias de perfurações estudadas.
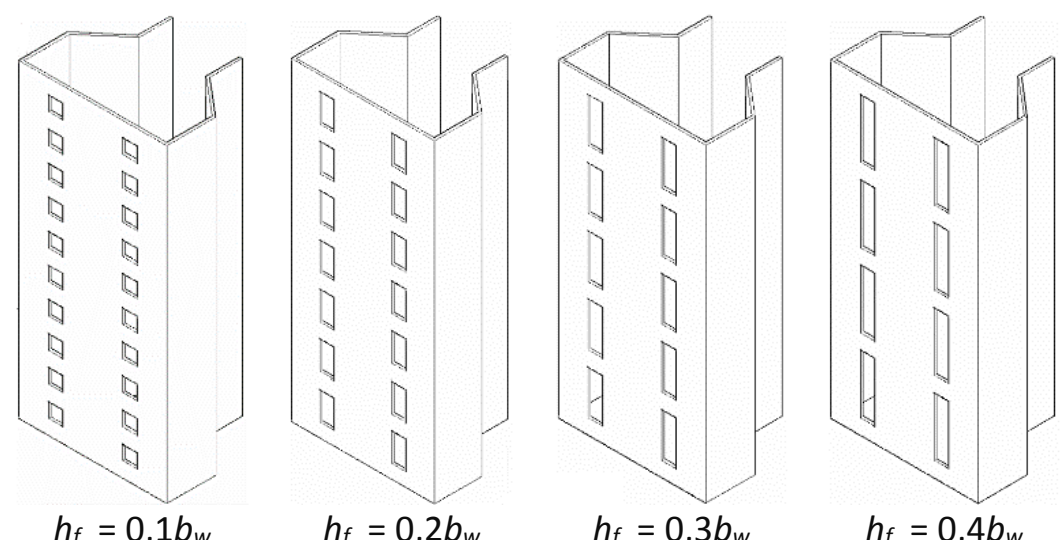

$$
h_{f}=0,3 b_{w}
$$$$
h_{f}=0,4 b_{w}
$$

Figura 7 - Tipologias de perfurações utilizadas nas análises numéricas.

\subsection{Modelo de elementos finitos}

O modelo numérico utilizado no ANSYS tem como base os trabalhos de Souza (2013) e Faria (2016). Tal modelo é composto por um pilar com seção do tipo rack, utilizando o elemento SHELL181 (Figura 8a), em conformidade com trabalhos anteriores de Freitas et. al (2010) e Souza (2013) em que foi realizada um estudo de vários elementos finitos de casca e melhores resultados foram encontrados quanto utilizado o SHELL181. Este elemento possui quatro nós com seis graus de liberdade (três translações e três rotações) em cada nó. 
A fim de que o empenamento da seção fosse restringido, foram modeladas chapas retangulares nas extremidades dos pilares. O centro geométrico (CG) das chapas coincidem com o CG do pilar. Estas chapas são representadas pelo elemento SOLID45 (Figura 8b), definido por oito nós, com três graus de liberdade (translações) em cada nó, com base nos estudos de Casafont et. al (2016) que preconizam a utilização do mesmo. As mesmas foram modeladas para se comportarem como elementos de rigidez elevada de modo que não ocorressem deformações nos níveis de carregamento utilizado.

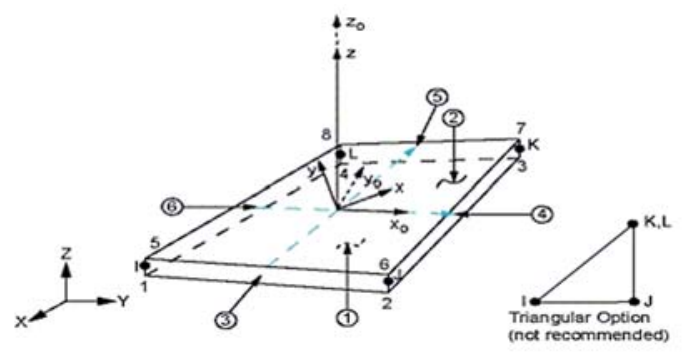

(a)
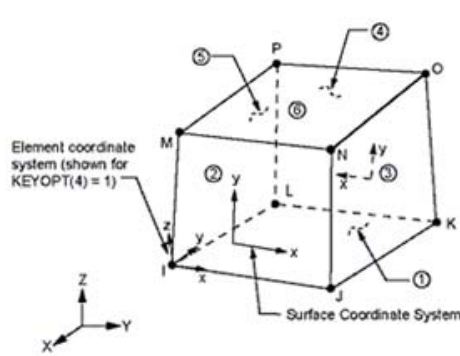

(b)

Figura 8 - Elementos utilizados: (a) SHELL181 e (b) SOLID45.

Em relação ao refinamento da malha, os elementos de superfície, isto é, os pilares, foram modelados utilizando um tamanho da malha igual a $10 \%$ da largura da alma da seção, conforme estudo de distribuição de malhas realizados nos trabalhos de Freitas et. al (2010), Souza (2013) e Faria (2016). Para os elementos de volume, utilizou-se uma malha mapeada, com volumes hexaédricos de largura também igual a 10\% de bw.

Para estabelecer a condição de extremidades rotuladas com empenamento restringido, em uma das chapas de extremidade, todos os graus de liberdade do nó correspondente à aplicação da força são restringidos a fim de simular as reações. Na outra chapa, no respectivo ponto, apenas os dois deslocamentos no plano da chapa são restringidos e aplica-se o carregamento pontual (Figura 9a). Para impedir a rotação do pilar em relação ao eixo longitudinal, foram inseridas duas restrições de deslocamentos (Figura 9b) em nós de cada uma das chapas de extremidade (Casafont, et al., 2016). Por fim, para garantir a restrição ao empenamento, os nós coincidentes da seção transversal do pilar com a chapa foram acoplados em todas as direções (Figura 9c). 


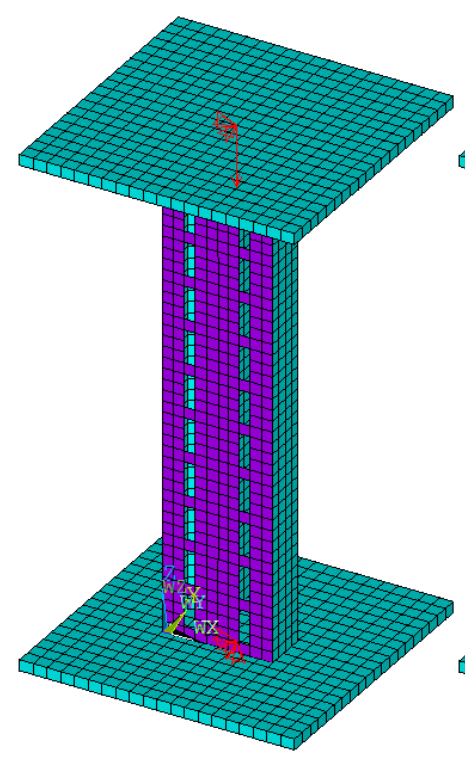

(a)

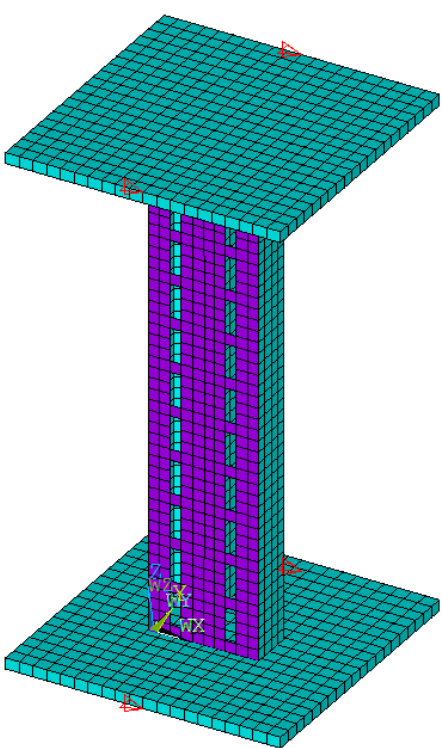

(b)

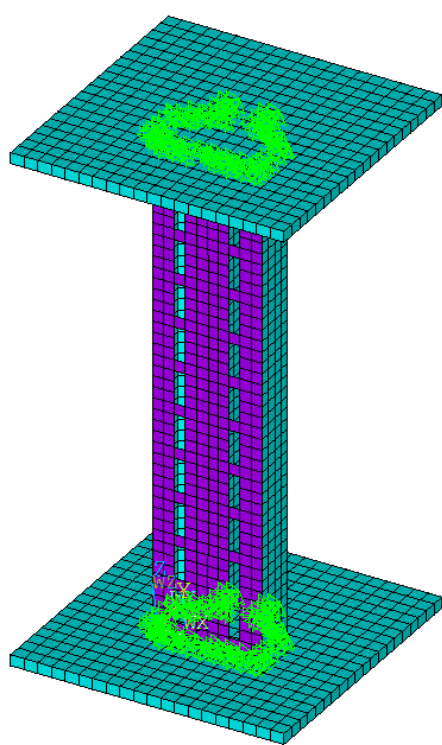

(c)

Figura 9 - Condições de contorno implementadas.

De forma a propiciar a automatização da construção do modelo, variação dos parâmetros e realização de análise paramétrica, foi utilizada a linguagem de programação do software ANSYS, a APDL (ANSYS Parametric Design Language).

Inicialmente foi realizada uma análise de estabilidade elástica da seção tipo rack submetida à compressão centrada, na qual é resolvido o problema de autovalor e autovetor, de forma a serem obtidas as forças críticas e os modos de instabilidade associados. Posteriormente, realiza-se uma análise não linear, em que as não linearidades física e geométrica são inseridas.

Para a consideração da não linearidade física foram inseridas as propriedades elastoplásticas do material, na qual utilizou-se um aço A36 $\left(f_{y}=250 \mathrm{MPa}\right.$ e $\left.f_{u}=400 \mathrm{MPa}\right)$, com um diagrama de tensão $x$ deformação multilinear (Faria, 2016). A não linearidade geométrica foi obtida atualizando a geometria dos pilares com a introdução de imperfeições iniciais baseadas no primeiro modo de instabilidade determinado na análise linear. A amplitude utilizada foi de 0,7 , correspondente à metade da menor espessura utilizada nas análises, no caso, 1,4 $\mathrm{mm}$. 


\subsection{Modelo pela Teoria Generalizada de Vigas}

Utilizando a Teoria Generalizada de Vigas (GBT) via programa GBTul, foram determinadas as forças críticas de instabilidade das seções estudadas. Como visto anteriormente, tal programa não permite a inserção de perfurações no modelo. Portanto, de forma a serem consideradas as perfurações, foi realizado procedimento proposto por Sales (2017), com base nos estudos de Moen e Schafer (2008).

Nos estudos de Sales (2017), foi proposta uma modificação à Equação 2.3 de Moen e Schafer (2008) para considerar o arranjo de furos fora dos limites apresentados nas Equações 2.2. A espessura fictícia da alma, $t_{f i c}$, foi tomada considerando-se a razão entre a largura efetiva, $b_{w, e f}$, e a largura da alma, $b_{w}$. Para o caso estudado, igual a $80 \%$. Além disso, as análises foram realizadas utilizando a Teoria Generalizada de Vigas, via GBTul.

$$
t_{f i c}=\frac{b_{w, e f}}{b_{w}} t_{w}=0,8 t_{w}
$$
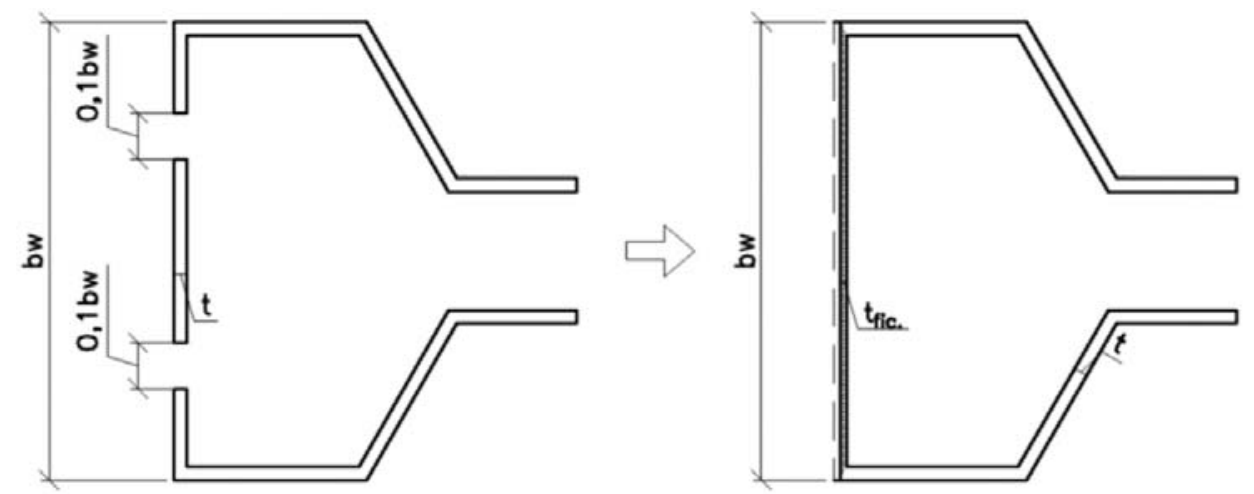

Figura 10 - Espessura fictícia da alma, $t_{\text {fic. }}$ (Fonte: Sales, 2017)

Assim, pode-se determinar as forças críticas de instabilidade distorcional considerandose as espessuras fictícias, $t_{\text {fic. }}$ Com esses valores é possível obter os valores de força última para os pilares das seções com perfurações.

\section{Resultados e discussões}

\subsection{Análise de instabilidade elástica das seções}

Na Figura 11 são apresentados os resultados das forças críticas de instabilidade, $P_{c r}$, obtidas no ANSYS para uma variação de comprimento, $L$, de até $3000 \mathrm{~mm}$, considerando 
o modelo com largura de alma igual a $80 \mathrm{~mm}$, em função das diferentes espessuras e tamanhos de furos estudados. As demais seções restantes apresentaram resultados semelhantes.

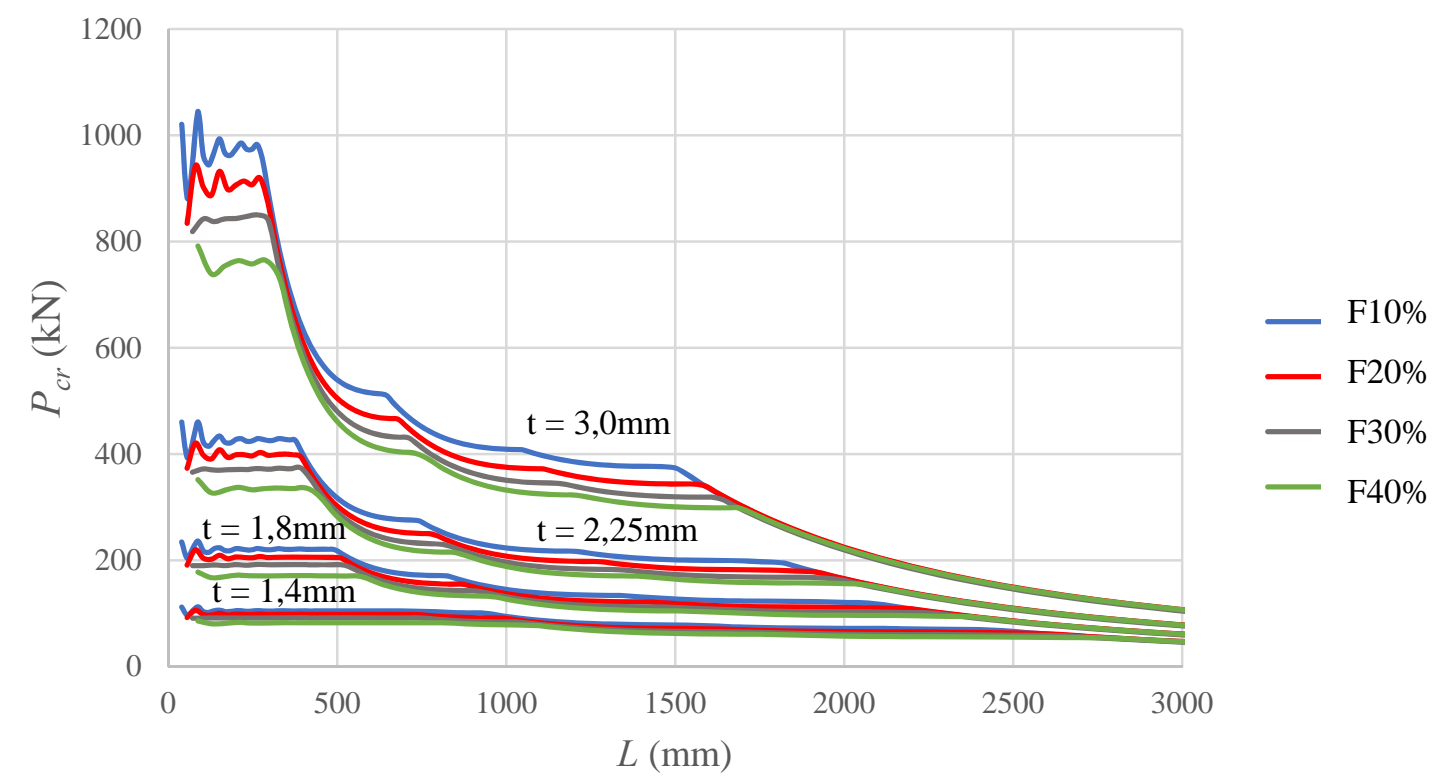

Figura 11 - Forças críticas obtidas no ANSYS para $b_{w}=80 \mathrm{~mm}$.

Como pode ser observado na Figura 11, percebe-se que a tendência do comportamento das curvas é similar e dependente da altura dos furos verticais, analisando uma mesma espessura. Para menores alturas de furos (F10\%), como esperado, a força crítica de instabilidade do pilar é maior. Observando cada gráfico separadamente e se mantendo a largura $b_{w}$ constante, pode ser observado que conforme se aumenta a espessura, a altura do furo influencia mais na dispersão dos valores das forças críticas. Isso é associado que com o aumento da espessura, a perda de área na seção transversal, para um mesmo tamanho de furo, torna-se mais evidente.

Observa-se que para pequenos comprimentos de pilar, há uma variação nos valores das forças críticas de instabilidade local. Presume-se que tal comportamento acontece em função da influência da proximidade da aplicação de força.

Na Figura 12 tem-se as curvas dos pilares com largura de $80 \mathrm{~mm}$ e espessura igual a 1,4 $\mathrm{mm}$, com a indicação dos pontos de mudança do modo de instabilidade predominante: local (L), distorcional (D) e global (G) e também os deslocamentos apresentados na seção transversal conforme o modo de instabilidade apresentado. 


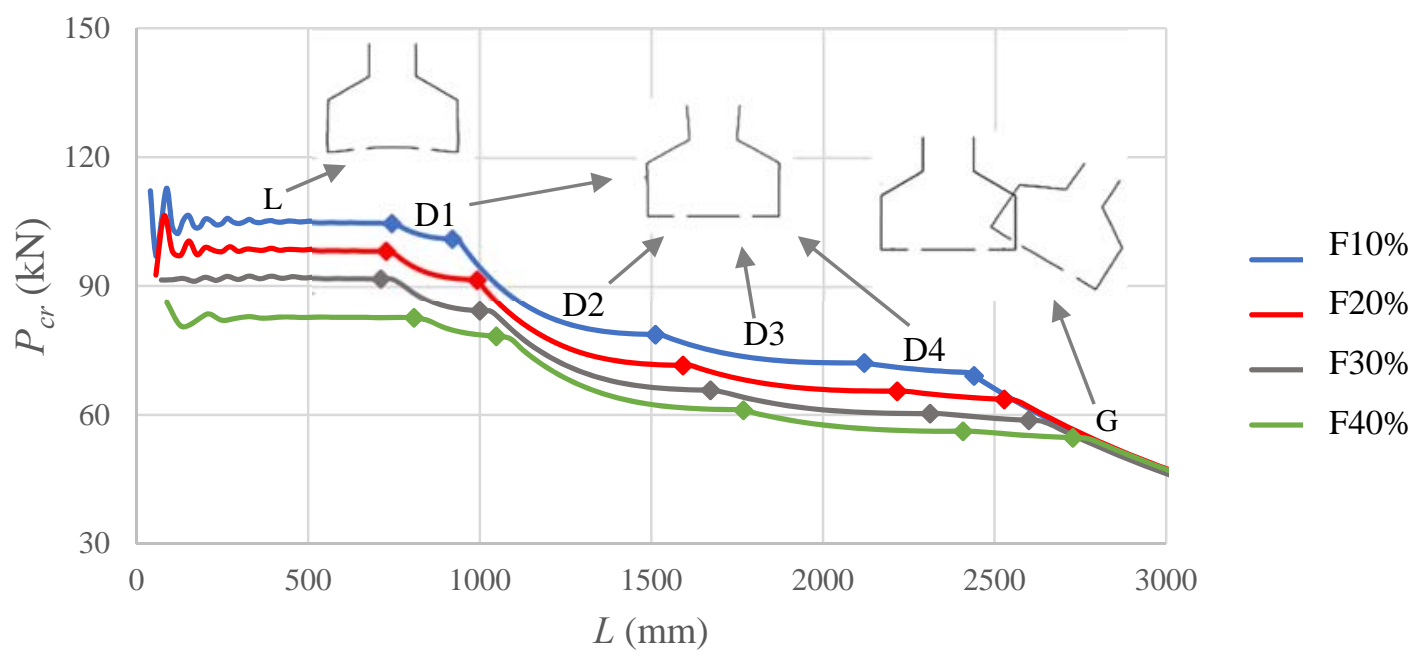

Figura 12 - Forças críticas obtidas no ANSYS para $b_{w}=80 \mathrm{~mm}$ e $t=1,4 \mathrm{~mm}$.

Da Figura 12 percebe-se que, ainda que a seção transversal não se altere em relação às quatro perfurações estudadas, as diferenças nas alturas dos furos influenciam na força crítica dos pilares. Além disso, estas seções apresentaram quatro modos distorcionais (D1, D2, D3 e D4), variando a quantidade de meias-ondas (Figura 13).

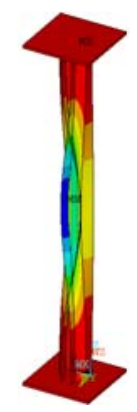

(a)

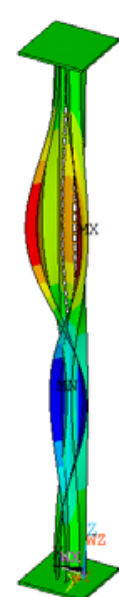

(b)

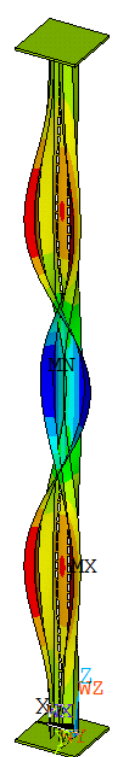

(c)

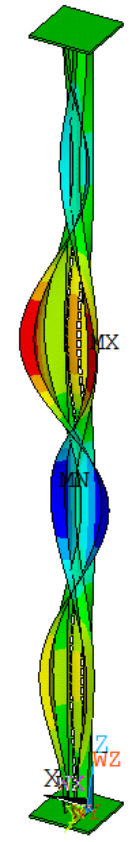

(d)

Figura 13 - Modos distorcionais: (a) D1, (b) D2, (c) D3 e (d) D4.

Utilizando a espessura fictícia da alma proposta por Sales (2017), Equação 3.1, foi realizada análise de estabilidade pelo GBTul das 16 seções transversais estudadas. 
Salienta-se que, as alturas de furo nessa análise não apresentam diferença, uma vez que possuem a mesma seção transversal. Na Figura 14 são apresentadas as curvas de instabilidade elástica para os modelos com largura de $80 \mathrm{~mm}$ e espessura igual a 1,8 mm, (analisados via ANSYS e com perfuração) e o mesmo modelo sem perfuração e com espessura fictícia, analisado via GBTul.

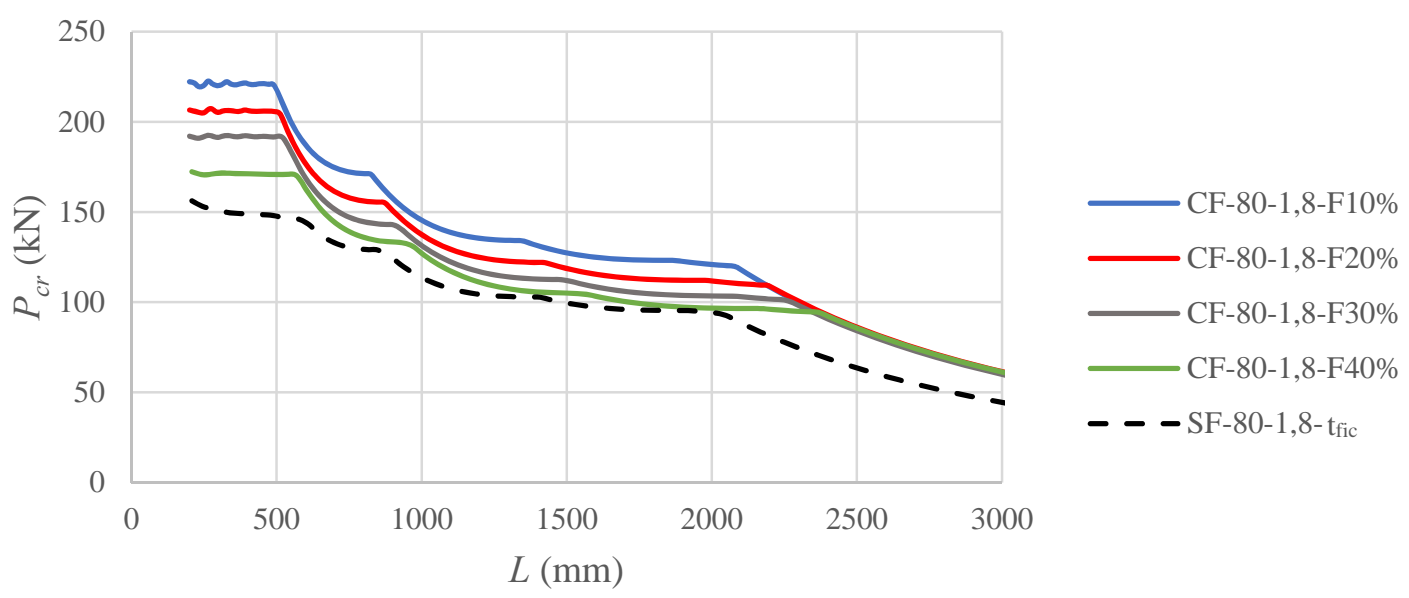

Figura 14 - Forças críticas obtidas no ANSYS e GBTul para modelos CF-80-1,8.

Percebe-se que as forças críticas obtidas considerando-se uma seção fictícia no GBTul apresentam a mesma tendência daquelas obtidas via ANSYS (com perfurações). Todavia, a mesma apresenta valores conservadores de forças críticas quando comparada aos furos de menores alturas. Comportamento semelhante foi observado para as outras seções.

\subsection{Análise de força última das seções}

Em seguida, foram obtidas as forças últimas, $P_{u}$, dos 64 modelos via ANSYS, com comprimentos variando de $500 \mathrm{~mm}$ a $1500 \mathrm{~mm}$, de modo a se evidenciar o modo de instabilidade distorcional. Na Figura 15 são apresentados os valores de força última versus comprimento para os modelos com $b_{w}=80 \mathrm{~mm}$ em função das diferentes espessuras e tamanhos de furos estudados. As demais seções restantes apresentaram resultados semelhantes. 


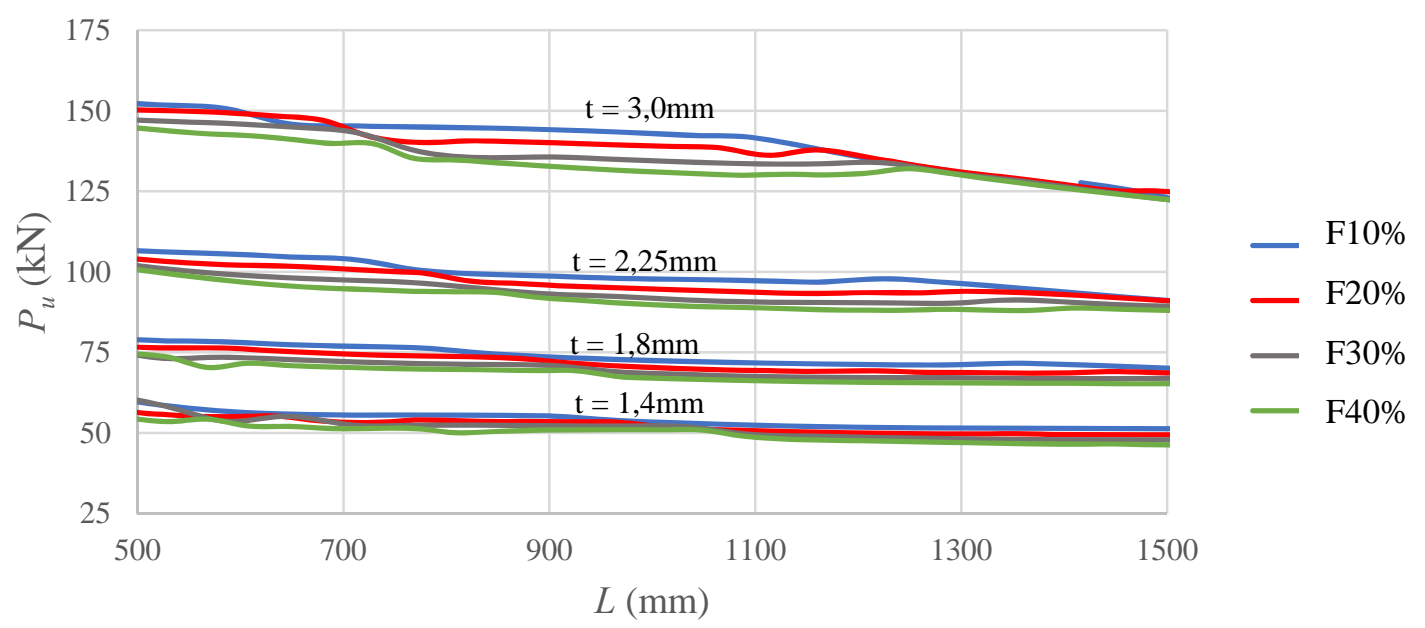

Figura 15 - Forças últimas obtidas no ANSYS para $b_{w}=80 \mathrm{~mm}$.

Percebe-se que a tendência do comportamento das curvas para a tipologia de furos estudadas é semelhante. Para menores alturas de furo, como esperado, a força última do pilar é maior. Observando cada gráfico separado e mantendo a largura $b_{w}$ constante, observa-se que, igualmente às forças críticas, conforme se aumenta espessura, a altura do furo influencia mais na dispersão dos valores de forças críticas.

Utilizando-se as forças críticas obtidas no GBTul com a espessura fictícia de alma em conjunto com as Equações 2.3 (MOEN), foram obtidas as forças últimas para essas seções (MOEN-tfic). A Figura 16 apresenta a comparação dos valores de força última obtidas via essa estratégia e via ANSYS para o modelo com $b_{w}=80 \mathrm{~mm}$ e $t=1,8 \mathrm{~mm}$.

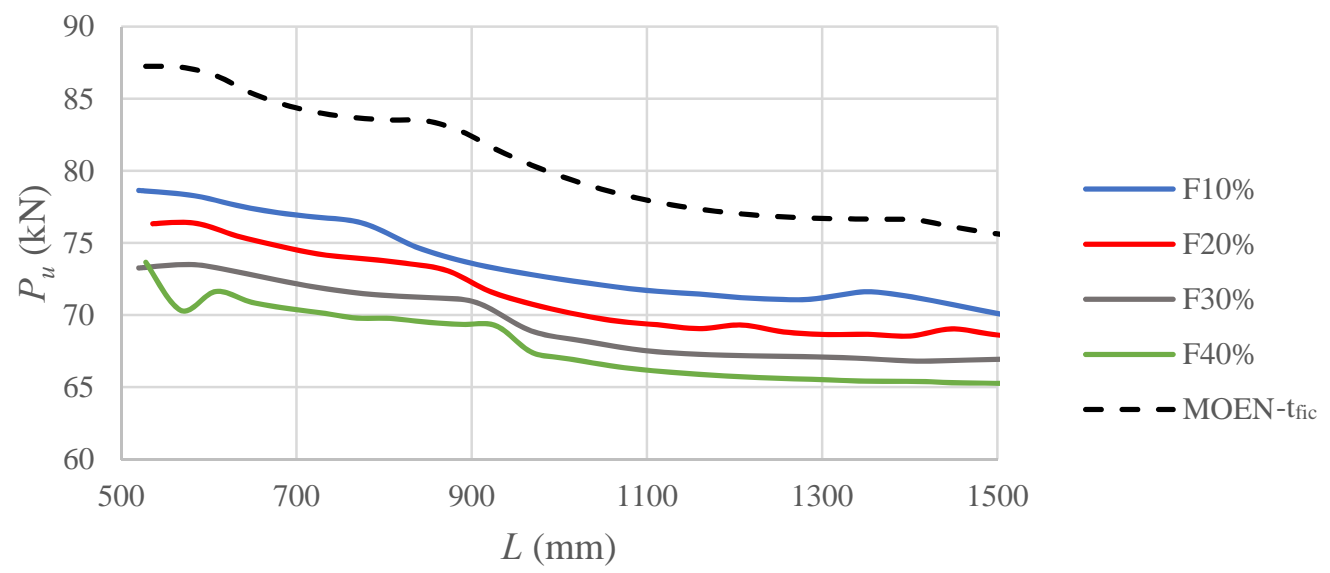

Figura 16 - Forças últimas obtidas no ANSYS e MOEN-t $t_{\text {fic }}$ para modelos 80-1,8. 
Analisando a Figura 16 percebe-se que a abordagem utilizando as Equações 2.3 (MOEN) e a espessura fictícia da alma de Sales (2017), não se mostraram adequadas à obtenção das forças últimas das seções com as tipologias de perfurações deste estudo. Presumese que isso ocorre pois nesse estudo os modelos apresentam uma maior densidade de furos quando comparados aos modelos estudados por Sales (2017), em que a estratégia se mostrou adequada.

\subsection{Comparações entre o MRD e os resultados numéricos do ANSYS}

A Figura 17 apresenta todos os resultados encontrados para as forças últimas via ANSYS para todas os modelos estudados, agrupados por largura de alma, $b_{w}$, e a curva de dimensionamento da ABNT NBR 14762:2010 para o modo distorcional (Equação 2.1).

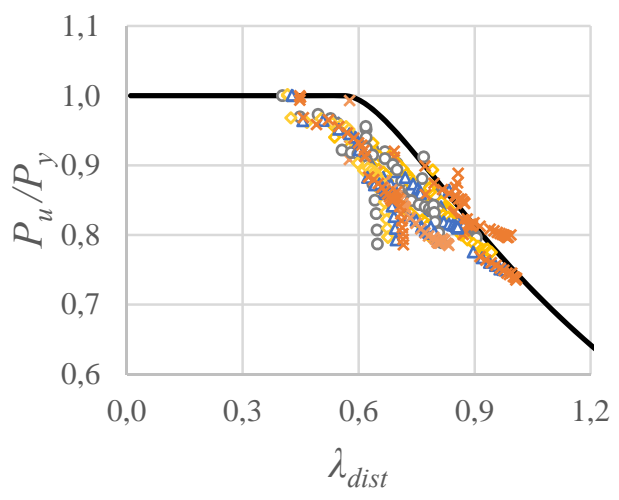

$b_{w}=80 \mathrm{~mm}$

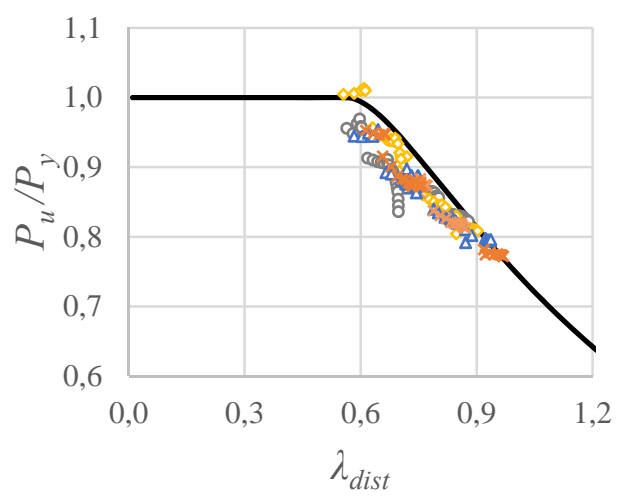

$b_{w}=110 \mathrm{~mm}$

$N_{c, \text { Rdist }} \quad$ o F10\%

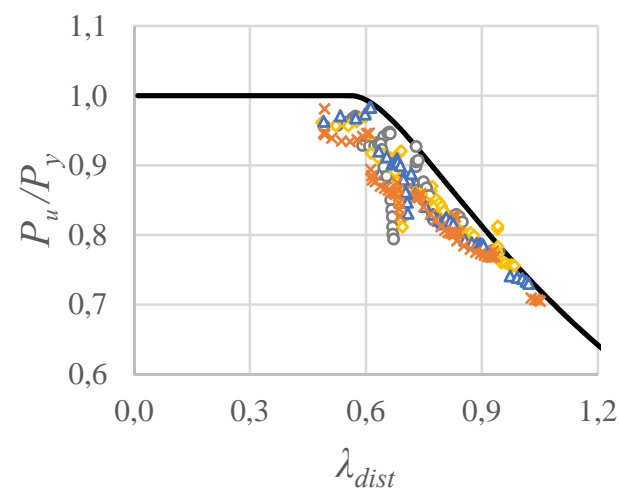

$b_{w}=94 \mathrm{~mm}$

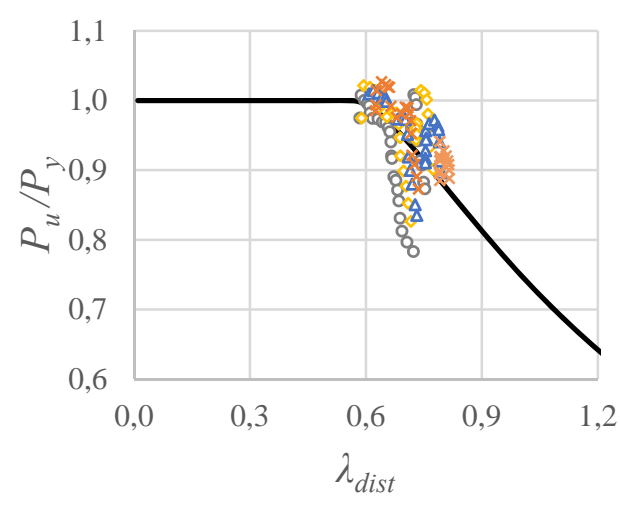

$b_{w}=120 \mathrm{~mm}$

$\diamond \mathrm{F} 20 \% \quad \triangle \mathrm{F} 30 \% \quad \times \mathrm{F} 40 \%$

Figura 17 - Comparação entre a curva da ABNT NBR 14762 e resultados numéricos. 
Como observado na Figura 17, conforme esperado, a prescrição normativa apresenta forças últimas maiores que os resultados numéricos, uma vez que a mesma não leva em consideração a existência de perfurações ao longo do elemento.

Na Figura 18 são destacados os resultados relativos ao modo distorcional do modelo CF$80-2,25$, com as quatro tipologias de perfurações estudadas.

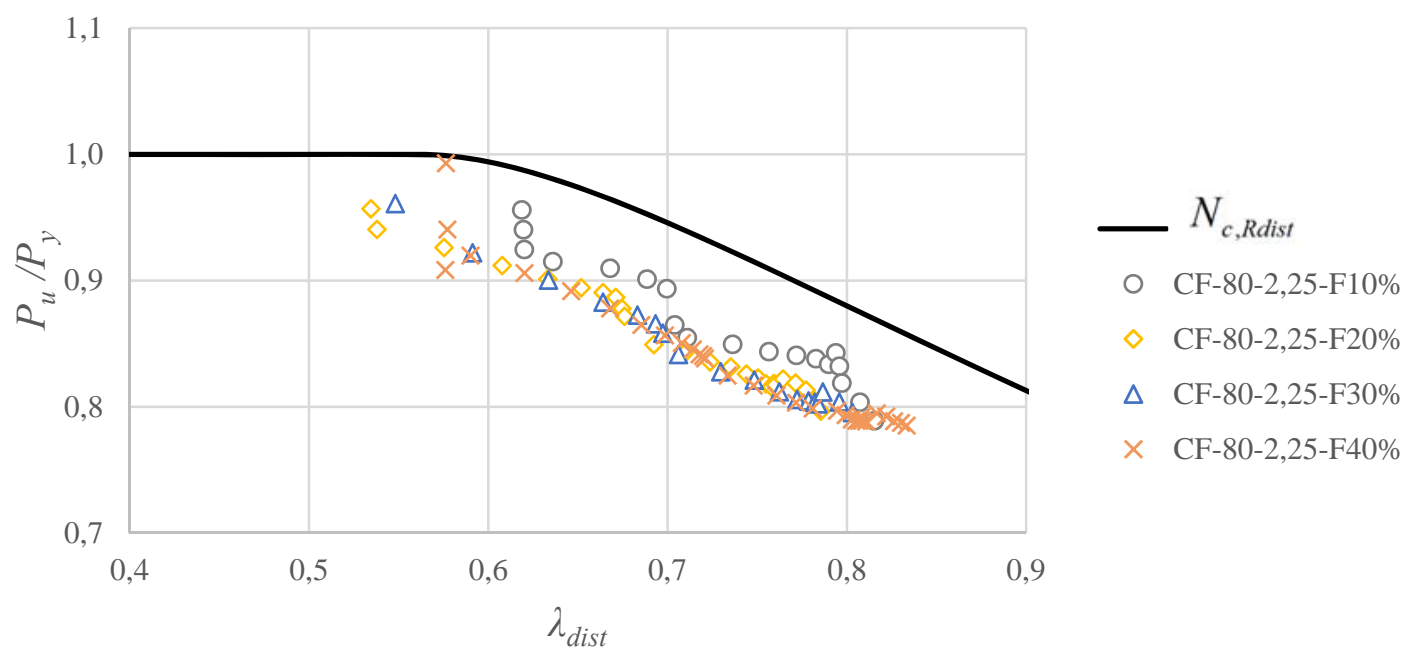

Figura 18 - Comparação entre a curva da ABNT NBR 14762 e forças últimas do ANSYS para o modelo CF-80-2,25.

Da Figura 18 percebe-se que os resultados numéricos indicam tendência similar à curva de dimensionamento segundo a prescrita na norma brasileira. Essa tendência indica a possibilidade de adequação da mesma curva para a consideração de seções que apresentam as perfurações previstas nas análises.

Portanto, torna-se necessário uma curva que apresente valores de forças últimas inferiores aos valores obtidos nas análises numéricas. Alterando-se os coeficientes da Equação 2.1, é possível obter uma curva que atenda aos resultados numéricos encontrados via ANSYS. Logo, propõe-se a seguinte modificação: 


$$
\begin{aligned}
& \lambda_{\text {dist, furo }} \leq 0,350 \rightarrow N_{c, \text { Rdist, furo }}=A_{\text {min, }, \text { iq }} f_{y} \\
& \lambda_{\text {dist }, \text { furo }}>0,350 \rightarrow N_{c, \text { Rdist }, \text { furo }}=\left(1-\frac{0,45}{\lambda_{\text {dist }, \text { furo }}^{0,4}}\right) \frac{A_{\mathrm{min}, \text { liq }} f_{y}}{\lambda_{\text {dist }, \text { furo }}^{1,1}} \\
& \lambda_{\text {dist, furo }}=\left(\frac{A_{\min , \text { liq }} f_{y}}{N_{\text {dist, furo }}}\right)^{0,5}
\end{aligned}
$$

Na Figura 19 são apresentadas as forças últimas para todas as seções estudadas, a curva original da ABNT NBR 14762:2010 e a nova curva adaptada para as perfurações estudadas.

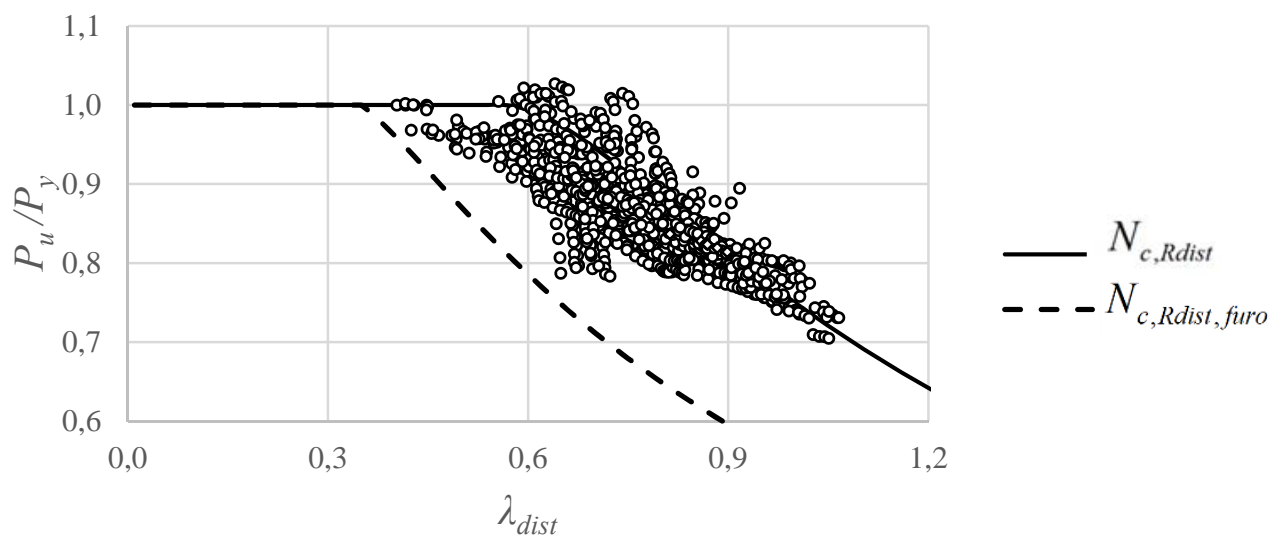

Figura 19 - Adequação da curva do modo distorcional da ABNT NBR 14762.

Pode-se perceber na Figura 19 que ao utilizar a Equação 4.1 a curva gerada apresenta valores de forças últimas inferiores aos resultados numéricos, podendo, portanto, ser utilizada no dimensionamento de seções com essa tipologia de furos.

\section{Considerações finais}

Observando-se os resultados numéricos do ANSYS, percebe-se que tanto em relação às forças críticas de instabilidade como às forças últimas, a tendência de comportamento dos resultados são similares. Como esperado, quanto maiores os tamanhos da perfuração, menores os valores encontrados (seja força crítica ou última).

As forças críticas de instabilidade foram obtidas via GBTul considerando uma espessura fictícia de alma, de forma a diminuir a rigidez da seção transversal em função das 
perfurações. De posse destas forças críticas, foram obtidas as forças últimas por meio de equações propostas por Moen e Schafer (2008). Os valores das forças últimas obtidas por essa estratégia não se mostraram adequados para o dimensionamento das perfurações estudadas. Presume-se que isso ocorreu pois nesse estudo os modelos apresentam uma maior densidade de furos quando comparados aos modelos estudados por Sales (2017), em que a estratégia se mostrou adequada.

Por fim, os resultados numéricos de forças últimas obtidos no ANSYS foram comparados com a curva de dimensionamento de instabilidade distorcional do Método da Resistência Direta da ABNT NBR 14762:2010. Como esperado, uma vez que a norma não considera a existência de perfurações, os resultados numéricos são inferiores aos prescritos. Portanto, foram propostas adequações à curva, de modo que a mesma pudesse ser utilizada no dimensionamento das seções e tipologias de perfurações estudadas.

\section{Agradecimentos}

Os autores agradecem à Universidade Federal de Ouro Preto (UFOP), FAPEMIG (Fundação de Amparo à Pesquisa do Estado de Minas Gerais), CNPq (Conselho Nacional de Pesquisa) e a CAPES (Coordenação de Aperfeiçoamento de Pessoal de Nível Superior).

\section{Referências bibliográficas}

ÁGUIA. Disponível em: <http://www.aguiasistemas.com.br/> Acesso em: 14 ago. 2017.

ANSYS, Ansys program version 17.0. Finite Element Software. Ansys Incorporation, Houston, PA, 2016

ASSOCIAÇÃO BRASILEIRA DE NORMAS TÉCNICAS. NBR 14762 - Dimensionamento de Estruturas de Aço Constituídas por Perfis Formados a Frio, Rio de Janeiro, 2010.

BATISTA, E. M. Effective section method: A general direct method for the design of steel coldformed members under local-global buckling interaction. Thin - Walled Structures 47, 345356, 2010.

BEBIANO R.; PINA P.; SILVESTRE N.; CAMOTIM D. GBTUL - Buckling and Vibration Analysis of Thin-Walled Members, DECivil/IST, Technical University of Lisbon, 2008.

CAMOTIM, D.; BASAGLIA C. On the behaviour, failure and direct strength design of thin-walled steel structural systems. Thin-Walled Structures, v. 81, p. 50-66, 2014. 
CASAFONT, M.; BONADA, J.; ROURE, F.; PASTOR, M. M. Selection of the initial geometrical imperfection in nonlinear FE analysis of cold-formed steel rack columns. Thin - Walled Structures 51, 99-11, 2012.

CASAFONT, M.; BONADA, J.; ROURE, F.; PASTOR, M. M. Imperfection amplitudes for nonlinear analysis of open thin-walled steel cross-sections used in rack column uprights. Thin - Walled Structures 76, 28-41, 2014.

CASAFONT, M.; BONADA, J.; ROURE, F.; PASTOR, M. M. Distortional Influence of Pallet Rack Uprights Subject to Combined Compression and Bending. Structures 8, 275-285, 2016.

CASAFONT, M.; MARIMON, F.; PASTOR, M.M. Calculation of pure distortional elastic buckling loads of members subject to compression via finite element method. Thin-Walled Structures 47, 701-729, 2009.

FARIA, V. O. Análise de estabilidade de perfis formados a frio com perfurações. Dissertação de Mestrado, PROPEC - UFOP, 2016.

FARIA, V. O. ; SOUZA, F. T. ; MIRANDA, S. A. ; SARMANHO, A. M. C. . Análise de perfis formados a frio com perfurações sob compressão centrada. Revista da Estrutura de Aço, v. 4, p. 163-180, 2015.

FREITAS, A. M. S.; FREITAS, M. S. R.; SOUZA, F. T. Theoretical analysis of perforated rack columns. SDSS Rio 2010 International Colloquium Stability and Ductility of Steel Structures v.2, p.953-960, 2010.

GODLEY, M. H. R. Storage Racking In Design of Cold Formed Steel Members. Chapter 11, Ed Rhodes. Elsevier Aplied Science, Amsterdam. pp. 361-399, 1991.

MOEN, C. D.; SCHAFER B. W. Direct strength design of cold-formed steel members with perforations. Research Report, The Johns Hopkins University, 2008.

NEIVA, L. H. A. Análise da influência da variação de perfurações no comportamento e capacidade de carga de perfis formados a frio de seção tipo rack. Dissertação de Mestrado, PROPEC - UFOP, 2017.

LANDESMANN, A.; CAMOTIM, D.; GARCIA, R. On the strength and DSM design of cold-formed steel web/flange-stiffened lipped channel columns buckling and failing in distortional modes. Thin-Walled Structures, v. 105, p. 248-265, 2016.

SALES, A. E. C. A. Análise da aplicação do método da resistência direta a perfis formados a frio com perfurações submetidos à compressão. Dissertação de Mestrado, MECOM - UFOP, 2017.

SARMANHO, A. M. C. Estudo do Comportamento Pós-Crítico de Paredes Esbeltas de Perfis Metálicos. Dissertação de Mestrado. COPPE - UFRJ, 1991.

SCHAFER, B. Designing cold-formed steel using the direct strenght method. 18th International Specialty Conference on Cold Formed Steel Structures, Orlando, Florida, 2006.

SCHAFER, B. Review: The Direct Strength Method of cold-formed steel member design. Journal of Constructional Steel Research 64, p.766-778, 2008.

SCHAFER, B., ÁDÁNI, S. Buckling analysis of cold-formed steel members using CUFSM: conventional and constrained methods. 18th International Specialty Conference on Cold Formed Steel Structures, Orlando, Florida, 2006.

SOUZA, F. T. Análise Teórico-Experimental da Estabilidade de Colunas Perfuradas em Perfis de Aço Formados a Frio em Seções Tipo Rack. Tese de Doutorado, PROPEC - UFOP, 2013.

ZHAO, X; REN C.; QIN, R. An experimental investigation into perforated and non-perforated steel storage rack uprights. Thin-Walled Structures, v. 112, p. 159-172, 2017. 